\title{
Política de indexação em ambiente colaborativo: uma análise em bibliotecas digitais brasileiras de teses e dissertações
}

Política de indización en ambiente colaborativo: un análisis en bibliotecas digitales brasileñas de tesis y disertaciones

Indexing policy in a collaborative environment: an analysis in Brazilian digital libraries of dissertations and thesis

\section{Roberta Cristina Dal' Evedove TARTAROttI, Paula Regina DaL' Evedove, Mariângela Spotti Lopes FUJITA}

(1) Universidade Estadual Paulista, Av. Hygino Muzzi Filho, 737, Campus Universitário, CEP. 17525900, Marília, SP, Brasil, roberta_tartarotti@yahoo.com.br, mariangelaslf57@gmail.com Universidade Federal de São Carlos, Av. Washington Luís, s/n, CEP. 13565-905, São Carlos, SP, Brasil, dalevedove@ufscar.br

\section{Resumen}

Uno de los desafíos del área de Organización y Representación del Conocimiento es acompañar la dinámica del tratamiento documental frente a los cambios tecnológicos y culturales experimentados en la contemporaneidad. Partiendo de la importancia de las directrices de la política de indización por materias en las bibliotecas digitales, el objetivo fue analizar la presencia de políticsa de indización en ambientes colaborativos frente a las especificidades inherentes que rodean a la indización social realizada por usuarios colaboradores para la representación temática de los documentos. Respecto a la metodología, se aplicó un cuestionario electrónico a los responsables de las bibliotecas digitales de tesis y disertaciones del Brasil. Los resultados señalan, en su mayor parte, la ausencia de una política de indización que ampare su práctica en ambientes colaborativos.

Palabras clave: Indización temática. Políticas de indización. Indización colaborativa. Bibliotecas digitales. Tesis y disertaciones. Brasil.

\section{Introdução}

Interesses e expectativas dos usuários em relação aos produtos e serviços de informação são cada vez mais complexos, urgentes e diversificados. Esta nova realidade vivenciada por bibliotecas e sistemas de informação do mundo todo é baseada nos preceitos da web 2.0, na qual práticas colaborativas nas ações de registro, organização e recuperação de objetos digitais foram viabilizadas. A interatividade em meio digital fomentou o surgimento e aplicação de processos de organização do conhecimento mais dinâmicos, rompendo com a tradicional aplicação de modelos seculares para a criação de produtos e serviços informacionais.

No tratamento dos documentos, uma das principais mudanças advém com a organização, representação e compartilhamento de informações e

\begin{abstract}
One of the challenges in the area of Knowledge Organization and Representation is to follow the dynamics of document treatment in face of the technological and cultural changes experienced in the contemporary world. Considering the importance of indexing policy guidelines for guiding indexing by subjects in digital libraries, the objective of this work was to analyze the presence of indexing policies in collaborative environments, facing the inherent specificities that surround the social indexing performed by users for the thematic representations of documents. Regarding the methodology, an electronic questionnaire was applied to the responsible persons fot the dissertations and thesis digital libraries in Brazil. The results indicate, mainly $i$, the absence of an indexing policy that supports the practice of indexing in collaborative environments.
\end{abstract}

Keywords: Indexing policy. Collaborative indexing. Digital libraries. Dissertations and thesis. Brazil.

objetos por usuários-colaboradores em ambientes digitais. Ainda que recente em termos teóricos e aplicações práticas, este novo sistema informal conhecido como indexação social ou folksonomia assevera o rompimento do modelo tradicional para o tratamento da informação e insere o usuário como tomador de decisão e parte ativa na descrição dos objetos digitais.

Em busca por informação de qualidade e compatível com a sua necessidade, o usuário atua como colaborador na realização da etiquetagem em recursos informacionais para o seu próprio uso, de acordo com os seus interesses e expectativas. Esta liberdade, fruto da inserção das Tecnologias de Informação e Comunicação nos sistemas de recuperação da informação, aliada às transformações culturais vivenciadas pelas sociedades pós-modernas, favorece uma descrição mais compatível com o contexto social dos usuários na 
condição de utilizadores, mediante atribuição livre de palavras-chave ou tags aos objetos e informações digitais.

Na representação social, os utilizadores escolhem e atribuem os seus próprios descritores para armazenar e recuperar conteúdos da Internet. Neste processo, tem-se a aproximação de indivíduos e comunidades com interesses comuns (Wal, 2006). De modo geral, a folksonomia é considerada (Damme 2006, p. 31)

[...] a social classification mechanism created by users which results in a bottom-up taxonomy. In this mechanism everyone can freely choose keywords for categorizing or describing their content. However, it is more than simply labeling or tagging objects or information, because this labeling process occurs in a social environment: everybody has access to the tagged content and the used tags and tag feedback, based on the input of others users, is generated.

A folksonomia altera substancialmente a maneira pela qual usuários e profissionais lidam com a informação. Surge como um recurso dinâmico e estratégico para categorizar e representar informações disponíveis na Web, sendo considerada um "[...] sistema democrático de tagging, que reflete a opinião do público em geral sobre determinado objeto, associado à identificação das pessoas que fazem essa classificação, introduzindo uma abordagem distribuída, inovadora, baseada em uma classificação social" (Vanderlei, 2006).

Apesar dos benefícios e do grande envolvimento de usuários e profissionais com a folksonomia, algumas questões sedimentadas nos âmbitos teórico e aplicado da área de Organização do Conhecimento ainda são trabalhadas de maneira tímida no ambiente digital, isso quando tais discussões existem. A política de indexação é uma destas temáticas que recebem pouca atenção da literatura especializada. O envolvimento de teóricos com as questões de política de indexação quase sempre está direcionado com os problemas inerentes aos sistemas tradicionais de recuperação da informação.

Conceitualmente, a política de indexação "é um guia de tomada de decisões" (Carneiro, 1985) e "a forma de realizar a indexação de uma determinada instituição, isto é, o esforço por concretizar, sistematizar e reunir em manuais os processos da indexação" (Gil Leiva, 2008). Caracteriza-se, ainda, como (Tartarotti, Dal' Evedove e Fujita, 2016, p. 9)

[...] um conjunto de diretrizes que favoreça o aprimoramento e a consistência na recuperação da informação. Por ser uma sistematização que integra a gestão da informação registrada, a política de indexação atua como recurso base para o estabelecimento de condutas teóricas e práticas dos profissionais envolvidos no tratamento da informação documentária.
Em estudo realizado por Rubi (2012) a autora apresenta, mediante revisão bibliográfica na literatura nacional e internacional de Biblioteconomia e Ciência da Informação, uma síntese de todos os elementos apontados por teóricos do tema como sendo importantes na elaboração de uma política de indexação correlacionando-os às suas fontes literárias, conforme consta no Quadro 1.

\begin{tabular}{|c|c|}
\hline $\begin{array}{l}\text { Elementos de política de } \\
\text { indexação }\end{array}$ & Fontes \\
\hline Avaliação do sistema & Carneiro (1985) \\
\hline $\begin{array}{l}\text { Campos e sub/campos do } \\
\text { formato MARC }\end{array}$ & Moen e Benardino (2003) \\
\hline Browsing & Fosket (1973) \\
\hline $\begin{array}{l}\text { Capacidade de revocação } \\
\text { e precisão do sistema }\end{array}$ & Carneiro (1985) \\
\hline Cobertura de assuntos & $\begin{array}{l}\text { Carneiro (1985); Guimarães } \\
(2000)\end{array}$ \\
\hline Consistência/ Uniformidade & $\begin{array}{l}\text { Olson e Boll (2001); Vílchez } \\
\text { Pardo (2002) }\end{array}$ \\
\hline $\begin{array}{l}\text { Detalhamento dos } \\
\text { procedimentos } \\
\text { relacionados à leitura } \\
\text { documentária }\end{array}$ & $\begin{array}{l}\text { Manuais INIS, AGRIS e } \\
\text { BIREME }\end{array}$ \\
\hline Economia & Vílchez Pardo (2002) \\
\hline Escolha da linguagem & $\begin{array}{l}\text { Carneiro (1985); Vílchez } \\
\text { Pardo (2002) }\end{array}$ \\
\hline Especificidade & $\begin{array}{l}\text { Cutter (1876); Foskett (1973); } \\
\text { Carneiro (1985); Chaumier } \\
\text { (1986); Connell (1996); Olson } \\
\text { e Boll (2001); Vílchez Pardo } \\
(2002)\end{array}$ \\
\hline Estratégia de busca & Carneiro (1985) \\
\hline Adequação & Olson e Boll (2001) \\
\hline Exaustividade & $\begin{array}{l}\text { Lancaster (1968; 1993); } \\
\text { Carneiro (1985); Chaumier } \\
\text { (1986); Van Slype (1991); } \\
\text { Olson e Boll (2001) }\end{array}$ \\
\hline $\begin{array}{l}\text { Forma de saída dos } \\
\text { resultados }\end{array}$ & Carneiro (1985) \\
\hline Formação do indexador & Fosket (1973) \\
\hline $\begin{array}{l}\text { Manual de indexação } \\
\text { (elaboração/utilização) }\end{array}$ & $\begin{array}{l}\text { Manuais INIS, AGRIS e } \\
\text { BIREME; Guimarães (2000) }\end{array}$ \\
\hline $\begin{array}{l}\text { Seleção e aquisição de } \\
\text { documentos-fonte }\end{array}$ & $\begin{array}{l}\text { Carneiro (1985); Guimarães } \\
(2000)\end{array}$ \\
\hline Síntese & Vílchez Pardo (2002) \\
\hline Uso (pelo usuário) & Vílchez Pardo (2002) \\
\hline
\end{tabular}

Quadro 1. Síntese dos elementos a serem considerados na elaboração de uma política de indexação (Rubi, 2012)

A aplicabilidade de uma política de indexação é de suma importância para uma melhor representação 
e recuperação temáticas em bibliotecas e sistemas de informação que utilizam a abordagem tradicional do processo de indexação realizada por bibliotecários. Contudo, percebemos que são incipientes estudos na literatura nacional de Biblioteconomia e Ciência da Informação sobre políticas de indexação para ambientes colaborativos, apesar da participação de usuários na representação temática dos objetos digitais ser uma realidade vivenciada no atual contexto biblioteconômico.

Ambientes sociais estão cada vez mais presentes no contexto de bibliotecas universitárias brasileiras, em que a livre criação de tags pessoais oferece uma solução para melhorar o acesso às informações, ao passo que são os próprios utilizadores que categorizam informações e objetos disponíveis em um ambiente aberto para a sua própria recuperação. Esta nova realidade experienciada em bibliotecas universitárias brasileiras sinaliza para a aplicação de princípios que solucionem os problemas e desafios advindos com as mudanças de representação e acesso à informação. Neste contexto, a política de indexação torna-se um instrumento que favorece a melhoria das condições de uso e aplicação da folksonomia, ao passo que orienta e direciona a representação de informações e objetos digitais conduzida por usuários, cuja participação neste processo ganha proporções jamais vistas.

Ao assumirem uma posição de tomador de decisão e parte ativa na descrição dos objetos digitais, nunca antes alcançada, a presença dos usuários em estudos e discussões acerca de políticas de indexação passa a ser indispensável, com especial atenção às questões próprias da indexação social praticada no ambiente colaborativo. Ao longo dos anos os estudos brasileiros sobre políticas de indexação conseguiram estabelecer uma base consistente para a elaboração, implantação e avaliação deste recurso para a representação e recuperação da informação em contexto de bibliotecas. As orientações envolvem fundamentos teóricos, metodológicos e pedagógicos que são facilmente aplicáveis na formação inicial e na formação continuada do bibliotecário indexador. Essa perspectiva pode ser observada nos estúdios de Carneiro (1985); Cervantes, Fujita e Rubi (2008); Dal'Evedove e Fujita (2015); Fujita e Santos (2016); Gil Leiva e Fujita (2012); Guimarães (2004); Lousada et al. (2011); Nunes (2004); Redigolo et al. (2012); Rubi (2008); Rubi, Fujita e Boccato (2012).

A política de indexação atua como sendo um recurso gerencial e estratégico do processo de indexação, uma vez que abarca um conjunto de decisões que refletem os interesses e objetivos de um sistema de informação em relação à representação e recuperação da informação. Por delimitar a cobertura temática em níveis qualitativos e quantitativos, imprime maior consistência à atividade de indexação.Na ótica de Fujita (2012, p. 22), a política de indexação é "um conjunto de procedimentos, materiais, normas e técnicas orientadas por decisões que refletem a prática e princípios teóricos da cultura organizacional de um sistema de informação". Constitui-se, portanto, um documento formal, estruturado e alinhado aos interesses do sistema de informação e de seus usuários que orienta as condutas teóricas e práticas do indexador. As diretrizes estabelecidas por cada instituição na busca pela consistência e qualidade da indexação são elaboradas com base em um conjunto de elementos que envolvem a cultura organizacional do sistema de informação e a sistematização do processo de indexação.

Apesar dos estudos na temática preconizarem sobre a necessidade do estabelecimento formal de uma política de indexação para a otimização do serviço e racionalização do processo de indexação, com vistas à melhoria da qualidade dos produtos e serviços oferecidos pelas bibliotecas, muitas instituições não formulam princípios e critérios considerados indispensáveis para a tomada de decisão por parte dos profissionais envolvidos com o processamento técnico. A inexistência de políticas de indexação é observada na maior parte das instituições do Brasil, assim como em países da América Latina (Fujita \& Gil Leiva, 2009).

Diante do cenário apresentado, a proposta neste estudo foi analisar a presença da política de indexação em ambientes colaborativos, frente às especificidades que cercam à indexação social realizada por usuários-colaboradores,, visto que estudos preocupados com este viés são incipientes no cenário nacional. Neste sentido, consideramos este um tópico interessante por discorrer sobre os contributos da política de indexação para a meIhoria e amparo das atividades de indexação social praticadas em ambientes colaborativos. Para tanto, elencamos as bibliotecas digitais de teses e dissertações de universidades públicas brasileiras como ambientes de análise e discussão dos elementos de política de indexação, em face de um maior nível de complexidade exigido para a adoção de normas e diretrizes que amparem a indexação social em ambiente colaborativo.

\section{Procedimentos metodológicos}

A fim de viabilizar o levantamento da políticas de indexação em bibliotecas digitais de teses e dissertações, os procedimentos metodológicos constaram de etapas subsequentes: levantamento das bibliotecas digitais existentes no Brasil; identificação do gestor responsável; e envio de questionário eletrônico. 
Na primeira etapa, foi realizada uma busca pelas bibliotecas digitais brasileiras de teses e dissertações a partir do site do IBICIT (Instituto Brasileiro de Informação em Ciência e Tecnologia), Órgão brasileiro responsável pela absorção e personalização de novas tecnologias, repassando-as as outras entidades interessadas na captura, distribuição e preservação da produção intelectual científica e tecnológica. Dentre outras iniciativas, o IBICT realiza a coleta automática de registro e disseminação de teses e dissertações de bibliotecas digitais do país, contribuindo para que o Brasil seja a quinta maior nação em número de repositórios digitais, por meio da Biblioteca Digital Brasileira de Teses e Dissertações (BDTD) (IBICIT, 2017).

No total, foram identificadas 106 instituições participantes da Rede BDTD, sendo 57 bibliotecas de teses e dissertações, 1 repositório comum e 48 repositórios institucionais, distribuídos em maior número na Região Sudeste do Brasil (39,62\%), seguida da Região Nordeste $(16,98 \%)$, da Região Sul (21,69\%), da Região Centro-Oeste $(9,44 \%)$ e da Região Norte, com apenas $4,72 \%$ deste tipo de sistema de recuperação da informação. Portanto, as bibliotecas digitais de teses e dissertações de instituições brasileiras estão presentes nas cinco regiões do país (Apêndice $A$ ).

A fim de identificar a presença de políticas de indexação nos ambientes identificados, com especial atenção à adoção da indexação social nestes sistemas de recuperação da informação, optamos pela elaboração e envio de um questionário eletrônico, sendo este composto de questões abertas e fechadas. Para oportunizar a coleta de dados junto às instituições contempladas na pesquisa, no primeiro momento realizamos a coleta dos e-mails dos gestores responsáveis disponíveis nos respectivos sites institucionais ou, em alguns casos, contato em formulário próprio. Neste momento, surgiram os principais obstáculos da pesquisa, ocasionados pelo não funcionamento adequado de alguns dos sites das bibliotecas digitais investigadas. Em alguns casos, o contato por telefone com o responsável pela Instituição foi necessário para que o envio do questionário fosse possível, sendo enviado em Agosto/2017. Cabe esclarecer que o prazo estipulado às Instituições para retorno das respostas foi de duas semanas. Após este período, foi enviado um lembrete para preenchimento do questionário eletrônico, prorrogando-se o prazo em mais uma semana. Em seguida, procedeu-se à análise dos dados.

No total, foram enviados 106 questionários eletronicamente, sendo obtidas 41 respostas de 33 diferentes instituições, o que corresponde a $31 \%$ das instituições identificadas.

\section{Resultados}

A pesquisa considera políticas de indexação para bibliotecas digitais, utilizando como exemplo bibliotecas digitais brasileiras. A primeira questão do questionário eletrônico teve como objetivo verificar a Instituição em que a biblioteca digital de teses e dissertações está vinculada. As demais questões foram subdivididas em: Política de Indexação nas Bibliotecas Digitais e Indexação social (folksonomia) nas Bibliotecas Digitais.

\subsection{Política de indexação nas Bibliotecas Digitais}

Nesta questão, buscamos levantar a adoção ou não de uma política de indexação nas bibliotecas digitais de teses e dissertações pesquisadas em nível nacional. De forma complementar, verificar, no caso de a instituição aplicar uma política de indexação, se a mesma está formalizada em um Manual de Indexação.

2. A Biblioteca Digital possui uma política de indexação que auxilie na atribuição dos assuntos das teses e dissertações da Instituição?

2.1 Se sim, está formalizada em um Manual de Indexação?

Constatamos que a maioria, ou seja, $63,3 \%$ das instituições contempladas na pesquisa não possuem política de indexação que norteie a atribuição dos assuntos de teses e dissertações, enquanto que em $36,6 \%$ das instituições a política de indexação está implantada. Para estas 12 Instituições que indicam possuírem uma política de indexação, observamos que apenas 4 das bibliotecas digitais possuem uma política de indexação formalizada em um Manual de Indexação, o que representa $33 \%$ do total.

Sim, manual do INIS , publicado pela International Atomic Energy Agency - IAEA Apropriado para a área nuclear ; quem realiza a indexação são bibliotecárias e pesquisadores da área, que trabalham na biblioteca com esta finalidade, e são devidamente treinados no uso do thesaurus do INIS. (Instituição A)

Além das palavras chaves utilizadas pelos alunos, atribuímos os assuntos estabelecidos campo (150) da Rede Bibliodata, e seguimos o Manual da Rede Bibliodata. (Instituição B)

A formalização da política em um Manual de indexação torna-se relevante ao ser um instrumento real de trabalho e norteador dos princípios do processo de tratamento documentário pela biblioteca, a fim de que seja garantida a consistência tanto na catalogação de assunto como na indexação (Tartarotti, 2014, p. 203). A continuidade e o aperfeiçoamento de uma política de indexação no decorrer dos anos dependem, sobretudo, 
de seu registro em documentos, "[...] de modo que se possa ter clareza do conjunto de decisões tomadas, suas razões e seu contexto" (Guimarães, 2000, p. 55-56). Não basta que os sistemas de informação tenham a compreensão clara dos procedimentos a serem seguidos no que compete à indexação dos recursos informacionais, mas é imprescindível que cada instituição registre formalmente as orientações a serem seguidas, estabelecendo parâmetros comuns a serem adotados por todos os bibliotecários.

\subsection{Indexação social nas Bibliotecas Digitais}

A terceira questão do questionário eletrônico teve como objetivo identificar a presença de elementos de folksonomia nas bibliotecas digitais de teses e dissertações:

3. A Biblioteca Digital permite a indexação colaborativa (folksonomia), ou seja, a participação dos usuários na atribuição dos assuntos das teses e dissertações?

\subsection{Se sim, explique.}

3.2 Se não, existe algum projeto para que este tipo de indexação seja adotada na Biblioteca Digital?

Referente à adoção da indexação social como prática de representação colaborativa nas bibliotecas digitais de teses e dissertações, $68,3 \%$ das instituições não adotam, enquanto que $31,7 \%$ afirmaram viabilizar, no sentido de permitir a abertura e sugestões de termos, assuntos ou descritores das teses e dissertações pelos próprios autores, em geral, durante a elaboração da ficha catalográfica dos trabalhos. Tais termos podem ser complementares aos vocabulários controlados adotados, que nem sempre alcançam o nível de especificidade necessário para a descrição temática de teses e dissertações:

Permite, mas não é necessário, os profissionais respondem bem pela indexação. (Instituição A)

Utilizamos as palavras chaves que os alunos adotam em suas teses, tanto em inglês como em português. (Instituição B)

São usadas somente as palavras-chave dos autores. (Instituição C)

Em alguns casos utilizamos as palavras-chave atribuídas pelo autor, no termo de autorização, por considerarmos que muitas vezes ele conhece termos mais consolidados na área de pesquisa dele. Considerando que o repositório não permite o uso de remissivas, inserimos o termo do vocabulário controlado e também o termo adotado na área. (Instituição D)

Existe a folksonomia nos materiais submetidos diretamente pelos autores. (Instituição E)

Em teses e dissertações os próprios acadêmicos inserem em seus resumos os termos que, de certa forma identificam e indexam este produto acadêmico. Nesta perspectiva o próprio usuário faz livremente a indexação, baseado é claro nos termos a qual o seu trabalho estiver vinculado e a área do conhecimento, que será identificado por seus pares e facilitará a recuperação e o compartilhamento dentro da área do conhecimento e atuação. (Instituição F)

Se necessário recorremos ao autor. (Instituição G)

Utilizamos também as palavras atribuídas pelos autores. (Instituição H)

É atribuída através da palavras-chave, informada pelos autores. Presente também através do autodepósito. (Instituição I)

Utilizamos muitas vezes as palavras-chaves atribuídas nos resumos/abstracts. (Instituição J)

Em parte, pois as bibliotecas setoriais levam em consideração as palavras-chave sugeridas pelos autores para a confecção da ficha catalográfica, o bibliotecário por sua vez, válida ou não este termo a depender dos instrumentos de validação, como thesaurus e vocabulários controlados. A atribuição dos assuntos para a comunidade de teses e dissertações, ocorre desta forma em nosso Repositório. (Instituição K)

Nas fichas catalográficas (feitas autormaticamente por um sistema de autopreenchimento) os alunos podem escolher até 5 assuntos. Quandos estas obras (teses ou dissertações) chegam a biblioteca, é feita uma comparação entre os assuntos atribuidos pelos autores e os existentes na base e em tesauros e vocabulários controlados (de acordo com o domínio do conhecimento). Quando são muito específicos e não existe equivalência em nenhum tesauro ou vocabulário controlodado, são adotados os assuntos utilizados pelos usuários. (Instituição L)

No entanto, embora as instituições investigadas consideram que esta prática tem consonância com as concepções da classificação colaborativa, na literatura a folksonomia em si é a atribuição realizada por leitores ou usuários destes trabalhos que não sejam os próprios autores, isto é, "folksonomia é o resultado da atribuição livre e pessoal de etiquetas às informações dos

recursos na Web, em um ambiente social, compartilhado e aberto a outros, pelos próprios

usuários da informação, visando a sua recuperação" (Catarino; Baptismo, 2007). Para tanto, seria necessário que as bibliotecas digitais de teses e dissertações permitissem mecanismos e políticas nestes sistemas de recuperação e políticas neste sentido.

Referente a projetos futuros nessa direção, a maioria das instituições não pretendem implementar a folksonomia. Entretanto, informações apresentadas indicam que melhorias estão sendo tomadas, o que favorece a abertura para os ambientes colaborativos: 
No momento estamos planejando elaborar uma política de indexação. (Instituição M)

Por enquanto ainda não, mas é algo que pretendemos elaborar. Infelizmente nós, assim como outras instituições temos sofrido com a falta de servidores, o que, muitas vezes nos impede de investir em planejamento, elaboração de políticas. (Instituição N)

Não sei se seria possível para outras áreas do conhecimento, no caso nuclear não há uso de linguagem natural para indexação. (Instituição O)

Como os usuários elaboram a ficha catalográfica pelo gerador automático de fichas pelo site da Instituição e atribuem as palavras-chave isso serve de uma certa forma, como orientação ou não para os bibliotecários, na elaboração dos assuntos. (Instituição P)

Existem planos futuros para o autoarquivamento. (Instituição Q)

A última questão do questionário favoreceu a reflexão dos sujeitos participantes da pesquisa acerca da inserção da indexação social em bibliotecas digitais. Os comentários e sugestões obtidos indicam que muitos profissionais reconhecem os benefícios da participação dos usuários nos serviços biblioteconômicos e do importante papel que a política de indexação exerce neste processo. Além disso, alguns profissionais investigados demonstram interesse para com o desenvolvimento de abordagens teóricas que tragam reflexões e contribuições acerca da constituição e elaboração de políticas de indexação para ambientes colaborativos.

É evidente a necessidade de melhorias na busca do nosso repositório, sobretudo quando não se tem uma política de indexação. Frente a esta necessidade, estamos em busca de ajuda para construir a nossa política. Caso possamos atuar de alguma forma, estamos receptivos a parcerias.

Estamos trabalhando para que a nossa base principal (pergamum) "converse" com a nossa BDTD para que os assuntos inseridos em um sejam também disponíveis na outra. A intenção é que na nossa BDTD tenha assuntos autorizados (do pergamum) e os livres (dos autores). Gostaríamos de receber informações de qualquer avanço que tenham nesta pesquisa.

\section{Considerações finais}

A política de indexação para e em ambiente colaborativo é um tema negligenciado pela literatura de Ciência da Informação, em especial no contexto brasileiro. Discussões que abordem o papel da política de indexação em ambiente colaborativo são oportunas para a ampliação de subsídios teóricos e melhor compreensão de seus efeitos no ambiente de bibliotecas digitais. Embora existam limitações nos resultados obtidos por conta do baixo número de respostas recebidas das Ins- tituições investigadas, a aplicação do questionário permitiu observar a presença da política de indexação nas bibliotecas digitais de teses e dissertações e a indicação de diretrizes e/ou aplicações práticas da indexação social nestes ambientes. Verificou-se ainda uma tendência de migração das teses e dissertações de bibliotecas digitais para repositórios institucionais, corroborando com a necessidade de diretrizes claras de política de indexação que norteiem tanto a representação como a recuperação temática nestes novos ambientes.

A condução desta pesquisa evidenciou que a maioria das bibliotecas digitais brasileiras de teses e dissertações ou repositórios institucionais analisados não possui uma política de indexação que ampare a prática contemporânea da indexação em ambientes colaborativos, isto é, não existem diretrizes ou normas direcionadas para os aspectos sociais e normativos da organização da informação neste contexto.

Como um fator-chave que influencia cada uma das etapas do processo de indexação, a elaboração, implantação e avaliação de uma política de indexação em um ambiente colaborativo pode permitir a melhoria da recuperação por assunto em bibliotecas digitais de teses e dissertações e em repositórios institucionais. Nesse sentido, consideramos essencial a construção contínua de diretrizes que satisfaçam todos os aspectos e sujeitos envolvidos neste processo, como princípio norteador, de modo a compor uma política de indexação que seja compatível com a realidade atual das bibliotecas digitais na era da representação colaborativa e busca em sistemas remotos de recuperação da informação por assunto.

De maneira complementar, estudos nesta abordagem são prementes no âmbito da Organização do Conhecimento, ao passo que propiciam amadurecimento da temática e contribuem para suprir lacunas conceituais e garantir diretrizes de política de indexação que sejam pautadas pelos elementos da indexação social. O compartilhamento de experiências práticas também favorece o desenvolvimento teórico da questão, cujas contribuições ampliam o entendimento dos requisitos e variáveis a serem consideradas neste novo formato de políticas de indexação que abarca orientações sobre o uso da folksonomia como forma de representação e recuperação de informação.

Sendo a representação colaborativa uma prática cada vez mais presente em bibliotecas, recomendamos novas alternativas de produção do conhecimento sobre política de indexação que sejam construídas coletivamente, tornando este recurso funcional e indispensável para ambientes 
colaborativos digitais. Neste processo, usuários devem compor grupos de trabalho que busquem discutir e formular os elementos que abarquem as especificidades da indexação social e do contexto de bibliotecas digitais e repositórios.

Complementarmente, este eixo investigativo deve ser observado sob a ótica dos repositórios digitais enquanto modelos de armazenamento e acesso de informações que ganham espaço em instituições acadêmicas e de pesquisa do mundo todo preocupadas com a melhoria da descrição dos recursos digitais e com as possibilidades de interatividade e autoarquivamento. Estudos dedicados à estruturação de diretrizes de política de indexação sob os preceitos da folksonomia devem ser ampliados de modo a amparar bibliotecas e repositórios digitais na tomada de decisões dos serviços bibliotecários em tempos de ações colaborativas, com o objetivo de sistematização do conhecimento sobre política de indexação para ambientes colaborativos.

\section{Notas}

(1) Disponivel em http://wiki.ibict.br/index.php/Bibliotecas_Digitais_de_Teses_e_Dissertações

\section{Apêndice A - Instituições participantes da Biblioteca Digital Brasileira de Teses e Dissertações (BDTD)}

Região Centro-Oeste

Banco Nacional de Desenvolvimento Econômico e Social (BNDS)

Centro de Ensino Unificado de Brasília

Companhia de Pesquisa de Recursos Minerais (CPRM)

Instituto Brasileiro de Informação Ciência e Tecnologia (IBICT)

Pontifícia Universidade Católica de Goiás (PUC-Goiás)

Universidade Católica de Brasília (UCB)

Universidade de Brasília (UnB)

Universidade Federal de Goiás (UFG)

Universidade Federal de Mato Grosso (UFMT)

Universidade Federal de Rondônia (UNIR)

Região Nordeste

Escola Bahiana de Medicina e Saúde Pública

Instituto Federal da Paraíba

Universidade Católica de Pernambuco (Unicap)

Universidade de Fortaleza (Unifor)

Universidade Federal de Pernambuco (UFPE)

Universidade Estadual da Paraíba (UEPB)

Universidade Estadual de Feira de Santana (UEFS)

Universidade Federal da Bahia (UFBA)

Universidade Federal da Paraíba (UFPB)

Universidade Federal de Alagoas (UFAL)

Universidade Federal de Campina Grande (UFCG)

Universidade Federal de Sergipe (UFS)

Universidade Federal do Ceará (UFC)

Universidade Federal do Pará (UFPA)

Universidade Federal do Rio Grande do Norte (UFRN)

Universidade Federal Rural de Pernambuco (UFRPE)

Universidade Federal Rural do Semi-Árido (UFERSA)

Universidade Salvador (UNIFACS)

\section{Região Norte}

Instituto Nacional de Pesquisas da Amazônia (INPA)

Universidade Federal do Amazonas (UFAM)

Universidade do Estado do Amazonas (UEA)

Universidade Federal de Roraima (UFRN)

Universidade Federal do Tocantins (UFT)

Região Sudeste
Centro de Desenvolvimento da Tecnologia Nuclear (CDTN)

Centro Universitário de Caratinga (UNEC)

Escola Superior de Propaganda e Marketing (ESPM)

Faculdade de Medicina de São José do Rio Preto (FAMERP)

Fundação Álvares Penteado (FECAP); Fundação Getulio Vargas (FGV)

Fundação João Pinheiro (FJP)

Fundação Oswaldo Cruz (Fiocruz)

Instituto de Engenharia Nuclear (IEN)

Instituto de Pesquisas Energéticas e Nucleares (IPEN)

Instituto Nacional de Pesquisas Espaciais (INPE)

Instituto Tecnológico de Aeronáutica (ITA)

Laboratório Nacional de Computação Científica (LNCC)

Pontifícia Universidade Católica de Campinas (PUC-Campinas)

Pontifícia Universidade Católica de São Paulo (PUC-SP)

Pontifícia Universidade Católica do Rio de Janeiro (PUC-Rio)

Universidade Anhembi Morumbi

Universidade Católica de Santos (UNISANTOS)

Universidade Federal de Juiz de Fora (UFJF)

Universidade de São Paulo (USP)

Universidade de Taubaté (UNITAU)

Universidade do Estado do Rio de Janeiro (UERJ)

Universidade do Grande Rio (Unigranrio)

Universidade do Oeste Paulista (Unoeste)

Universidade Estadual de Campinas (UNICAMP)

Universidade Federal do ABC

Universidade Federal do Triângulo Mineiro (UFTM)

Universidade Estadual Paulista (UNESP)

Universidade Federal de Minas Gerais (UFMG)

Universidade Federal de Ouro Preto (UFOP)

Universidade Federal de São Carlos (UFSCar)

Universidade Federal de São Paulo (Unifesp)

Universidade Federal de Uberlândia (UFU)

Universidade Federal de Viçosa (UFV)

Universidade Federal do Espírito Santo (UFES)

Universidade Federal do Rio de Janeiro (UFRJ)

Universidade Federal Fluminense (UFF)

Universidade Universidade do Sagrado Coração (USC)

José do Rosário Vellano (UNIFENAS)

Universidade Metodista de São Paulo (UMESP)

Universidade Nove de Julho (UNINOVE)

Universidade Presbiteriana Mackenzie

Região Sul

Faculdades EST

Pontifícia Universidade Católica do Paraná (PUC-PR)

Pontifícia Universidade Católica do Rio Grande do Sul (PUCRS)

Centro Universitário Univates; Universidade Católica de Pelotas (UCPel) Universidade de Caxias do Sul (UCS)

Universidade do Estado de Santa Catarina (UDESC)

Universidade do Vale do Rio dos Sinos (Unisinos); Universidade Estadual de Londrina (UEL); Universidade Estadual de Ponta Grossa (UEPG)

Universidade Estadual do Oeste do Paraná (UNIOESTE)

Universidade Federal da Fronteira Sul (UFSS)

Universidade Federal da Integração Latino-Americana (UNILA)

Universidade Federal de Pelotas

Universidade Federal de Santa Catarina

Universidade Federal de Santa Maria (UFSM)

Universidade Federal do Pampa (UNIPAMPA)

Universidade Federal do Paraná (UFPR)

Universidade Federal do Rio Grande (FURG)

Universidade Federal do Rio Grande do Sul (UFRGS)

Universidade Regional de Blumenau (FURB)

Universidade Tecnológica Federal do Paraná (UTFPR)

Universidade Tuiuti do Paraná (UTP)

\section{Referências}

BIREME; Centro Latino-Americano e do Caribe de Informação em Ciências da Saúde; Organização Mundial da Saúde; Organização Panamericana da Saúde; Organização Mundial da Saúde (Brasil) (2008). Manual de indexação de documentos para a base de dados LILACS. 2nd ed. São Paulo: BIREME/OPAS/OMS.

BIREME; Centro Latino-Americano e do Caribe de Informação em Ciências da Saúde; Organização Mundial da Saúde; Organização Panamericana da Saúde; Organização Mundial da Saúde (Brasil) (1988). Manual de indexação de documentos para a base de dados LILACS. São Paulo: BIREME. 
Carneiro, M. V. (1985). Diretrizes para uma política de indexação. // Revista da Escola de Biblioteconomia da UFMG $14: 2,221-241$.

Cervantes, B. M. N.; Fujita, M. S. L.; Rubi, M. P. (2008). Terminologias em política de indexação. // Advances y perspectivas en sistemas de información y documentación. Zaragoza: Universidad de Zaragoza. 211-222.

Chaumier, J. (1986). Analisis y lenguajes documentales. Barcelona: Mitre.

Connell, T. H. (1996). Subject cataloging. // Smith, L. C.; Carter, R. C. (Eds.). Technical services management, 1965-1990: a quarter century of change and a look to the future. New York: Haworth Press. 211-222.

Cutter, C. A. (1876). Rules for a printed dictionary catalogue. Washington, D.C.: Government Printing Office.

Dal'Evedove, P. R; Fujita, M. S. L. (2015). A pesquisa sobre indexação no Brasil: avanços e desafios. // Scire. 17:2, 49-56.

Damme, C. V. (2006). Folksonomies and enterprise folksonomies. Faculty of Economic, Political and Social Science and Solvay Management School.

Foskett, A. C. (1973). A abordagem temática da informação. Translated by Antonio Agenor Briquet de Lemos. São Paulo: Polígono; Brasília: UnB.

Fujita, M. S. L. (2012). A política de indexação para representação e recuperação da informação. // Política de indexação. São Paulo; Marília: Cultura Acadêmica; Oficina Universitária. 17-30.

Fujita, M. S. L.; Gil Leiva. (2009). Política de indexação na América Latina. // Ibersid: revista de sistemas de información y documentación. Zaragoza: Universidad de Zaragoza. 155-162.

Fujita, M. S. L.; Santos, L. P. (2016). Política de indexação em bibliotecas universitárias: estudo diagnóstico e analítico com pesquisa participante. // Transinformação. 28:1, 59-76.
Gil Leiva, I.; Fujita, M. S. L. (Orgs.) (2012). Política de indexação. São Paulo: Cultura Acadêmica; Oficina Universitária.

Gil Leiva, I. (2008). Manual de indización: teoría y práctica. Gijón: Trea.

Guimarães, J. A. C. (2004). As políticas de indexação como elemento para a gestão do conhecimento nas organizações. // Tecnologia e conteúdos informacionais: abordagens teóricas e práticas. São Paulo: Polis. 43-52.

Guimarães, J. A. C. (2000). Políticas de análisis y representación de contenido para la gestión del conocimiento en las organizaciones. // Scire. 6:2, 48-58.

Lancaster, F. W. (1993). Indexação e resumos: teoria e prática. Translated by Antonio Agenor Briquet de Lemos. Brasília: Briquet de Lemos.

Lancaster, F. W. (1968). Information retrieval systems: characteristics, testing and evaluation. New York: John Wiley \& Sons.

Lousada, M. et. al. (2011). Políticas de indexação no âmbito da gestão do conhecimento organizacional. // Informação \& Sociedade. 21:1, 191-202.

Moen, W. E.; Benardino, P. (2003). Assessing metadata utilization: an analysis of MARC content designation use. // Dublin Core Conference: supporting communities of discourse and practice: metadata research and application. Seattle. Online Proceedings. Seattle: Information School of the University of Washington.

Nunes, C. O. (2004). Algumas considerações acerca da ausência de políticas de indexação em bibliotecas brasileiras. // Biblos. 16: 55-61.

Olson, H. A.; Boll, J. (2001). Subject analysis in online catalogs. 2nd ed. Englewood, CO: Libraries Unlimited.

Redigolo, F. M. et. al. (2012). Elementos de política de indización para bibliotecas universitarias del área médica. // Scire. 18, 75-86.

Enviado: 2018-04-13. Segunda versión: 2018-06-19 Aceptado: 2018-06-24. 